\title{
LOS and NLOS Channel Models for Indoor 300 $\mathrm{GHz}$ Communications
}

\author{
Joonas Kokkoniemi, Janne Lehtomäki, and Markku Juntti \\ Centre for Wireless Communications (CWC), \\ University of Oulu, P.O. Box 4500, 90014 Oulu, Finland \\ Email: joonas.kokkoniemi@oulu.fi
}

\begin{abstract}
This paper studies the impact of the line-of-sight (LOS) and non-LOS (NLOS) path loss at $300 \mathrm{GHz}$ frequency band in a multipath propagation setting. The aim is to model the impact of the multipath signal components in stochastic indoor environment as a function of antenna patterns, and the number of the multipath signal components. The outcome of simulation model is that the general free space path loss is an accurate measure of the propagation loss in the LOS case. In the NLOS cases, the free space path loss model remains accurate with slight increase in the path loss exponent from 2 to approximately $\mathbf{2 . 2}$ to 2.7 depending on the material properties. Such low path loss exponents are caused by highly directional antennas omitting the most the interference from the propagation environment. As a consequence, the channel appears as a free space channel with some additional loss caused by reflections in NLOS directions.
\end{abstract}

\section{INTRODUCTION}

The millimeter wave frequencies (mmWave, 30-300 GHz) and beyond are in the focal point of the future cellular systems' development. These frequencies offer potential spectrum for ultra-high data rate communications because of the vast available frequency resources. Especially the $\mathrm{THz}$ frequency band $(300 \mathrm{GHz}-10 \mathrm{THz})$ has been subject to intensive academic research over the past decade [1]. The high path loss in the channel makes the mmWave and $\mathrm{THz}$ frequencies mostly suitable for short range communications. As the future networks' research aims at increasing the capacity by network densification, these extremely high frequencies sit in the future wireless ecosystem perfectly. The potential applications vary from massive user densification to extremely high data rate data kiosk applications and to wireless backhauls [2].

One of the specific application of interest for the $\mathrm{THz}$ frequencies are the indoor communication scenarios. For instance, high speed $\mathrm{THz}$ access points that provide huge local capacity inside a room or an office [3]. In-room communications has an advantage of inherently short communication distance, but also very minimal interference to adjacent cells due to very large penetration losses at the $\mathrm{THz}$ band [4] However, even at short distances high antenna gains are required leading to challenges in finding the users and devices, as well as beam tracking of moving devices.

This paper focuses on statistical modeling of the indoor THz propagation by Monte Carlo simulations assuming perfect antenna alignment. The advantage of the high frequency, high antenna gain systems is the ability to isolate the propagation phenomena and stack those to theoretical models that usually perfectly fit with the respective measurements. There are many papers about $\mathrm{THz}$ indoor channel and propagation modeling by measurements, simulations, and theoretical works, such as [5]-[11]. The approach of this paper differs from the existing work in a sense that we create nearly perfectly random indoor channel by applying distributions for potential objects in the indoor space. Therefore, we can introduce random reflection points representing objects in the environment, such as furniture. We also model certain deterministic reflection points from the walls, floor and ceiling along with the lineof-sight (LOS) path between the transmitter (Tx) and receiver (Rx). By utilizing Monte Carlo simulations in random indoor environment gives some insights into a $\mathrm{THz}$ band system operation in generic indoor locations. All rooms differ a bit, although usually sharing certain features, such as furniture placement in certain types of rooms, like living rooms. The above mentioned random reflection points can be anywhere in the room and ultimately the antenna patterns thin the number of these within the environment as the higher is the gain, the narrower the radiation pattern of the antenna is.

We derive channel models for LOS case, i.e., when the primary communication path is the LOS path and the non-LOS (NLOS) paths add power to the main channel. The second channel model is a NLOS model, where one of the NLOS paths is the primary communications channel and the rest of the NLOS paths again sum to the primary channel. We show that path loss of these NLOS models depend on the material characteristics of the environment. The LOS channel is less sensitive due to dominating best-case scenario propagation path. The simulated channel models in this paper focus on the $300 \mathrm{GHz}$ center frequency.

The rest of this paper is organized as follows. Section II gives the simulation model, Section III derives the simulated path loss models, and Section IV concludes the paper.

\section{Simulation Model}

The simulation model utilized to study the signal multipath propagation in indoor locations is a rectangular space limited by the walls, floor, and ceiling. An illustration of the the assumed system is given in figures 1 and 2 . The dark centered diamonds are transmitters (Txs) and the by placing those in tight grid across the room gives a general overview of the path loss from any location in the room. Those also represent wide range of possible communication distances between the $\mathrm{Tx}$ 


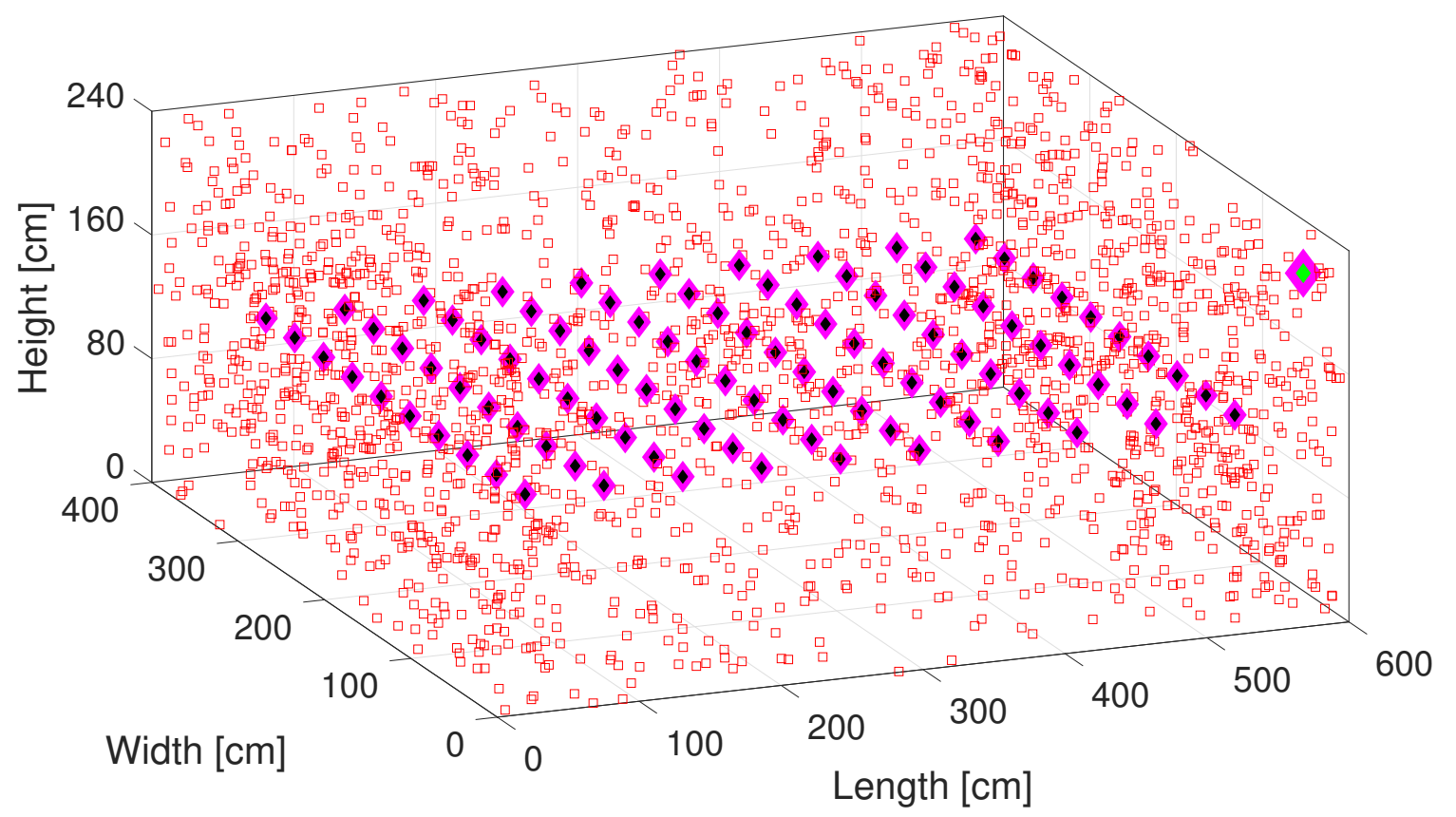

Fig. 1. An illustration of the simulation environment. Dark centered diamonds depict the Tx grid, green centered diamond in the corner depicts an access point, or the Rx. The red squares are random reflection points representing objects in the environment.

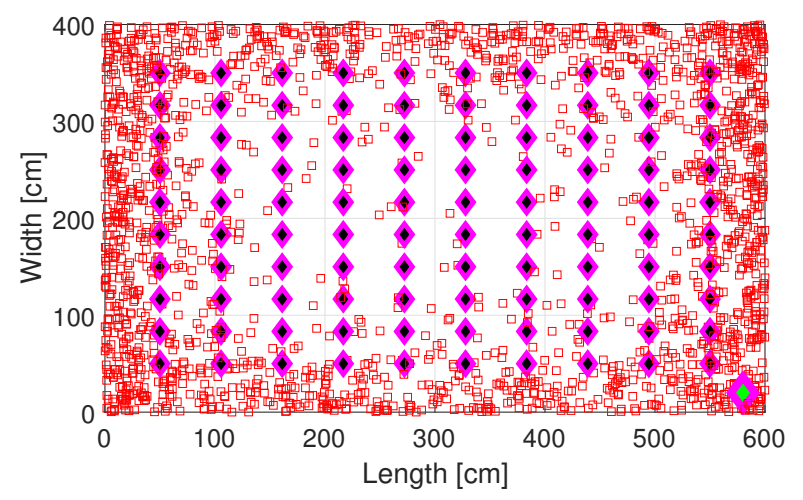

Fig. 2. View of Fig. 1 from above showing the distribution of the random reflection points.

and the Rx. The single green centered diamond is the receiver $(\mathrm{Rx})$. The $\mathrm{Rx}$ is assumed to be in the upper corner of the room, $20 \times 20 \times 20 \mathrm{~cm}^{3}$ away from top corner. It acts as a access point with good visibility to the room and to the Txs that are possible transmitting device positions.

Given a position of a particular Tx with respect to the $\mathrm{Rx}$, the walls act as potential reflecting surface for multipath propagation of the transmitted signals. The red squares in the figures are random reflecting points caused, e.g., by people, furniture, and other irregularities in the room. Those are modeled by exponential distribution with $40 \mathrm{~cm}$ mean to emphasize the fact that the most of the furniture and other objects are usually placed close to the walls. Therefore, it is less likely that there would be reflection sources in the center of the room.

In the Monte Carlo simulations the random reflection points and the number of them are picked randomly all around the room. The walls cause a deterministic reflection points as there is one single possible reflection point for each wall, and one for both the ceiling and the floor, dependent on the positions of the Rx and Tx. It is highly unlikely that all the deterministic reflection points would be available. Therefore, certain blocking probability is employed for each of these paths. Finally, all the available random and deterministic paths are summed at the receiver that causes constructive and destructive summation based on the phase of the signals arriving through different paths.

Finally, the availability of the randomly selected reflection points and the deterministic reflection points is dependent on the selected antenna pattern and the position of the Rx and Tx. As an example, isotropic antennas see the entire space and therefore all the reflection points. Very highly directional antennas would only see each other and with very high certainty, no reflection points at all. However, the antenna gain patterns are utilized to calculate the gains towards all reflection points. We consider two scenarios in the numerical results, one with the LOS available, and one where the primary communication path is selected among the reflection points by steering the Rx and Tx antennas towards that reflection point.

Given the random and deterministic paths of the multipath 


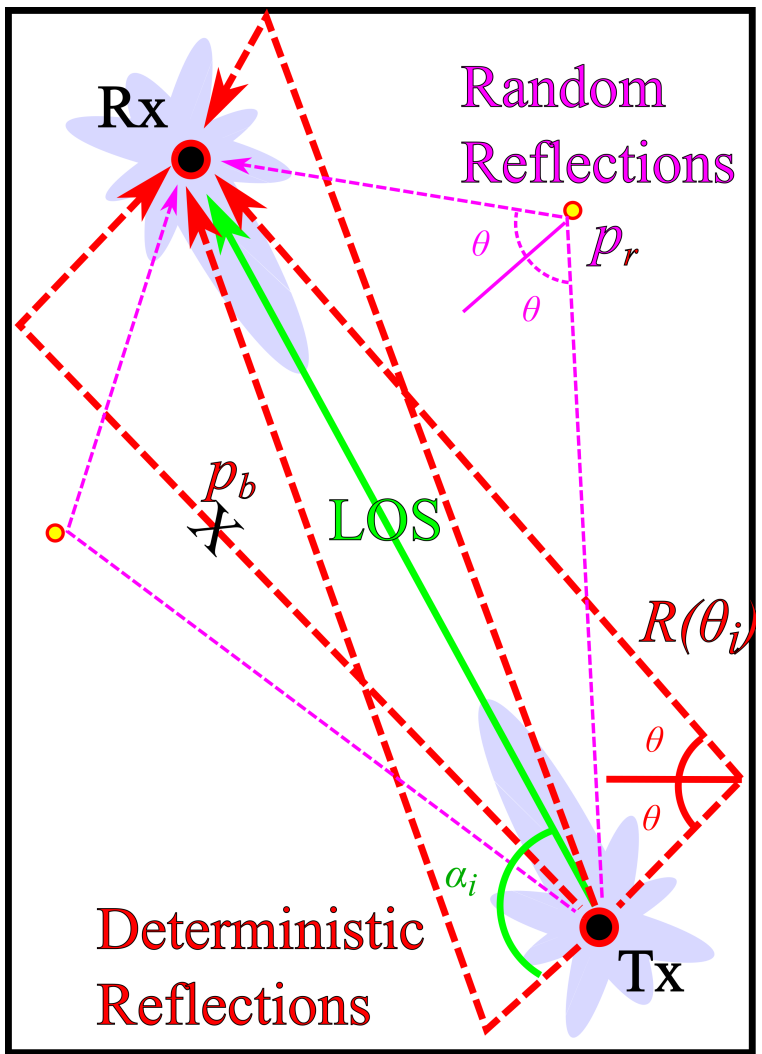

Fig. 3. Illustration of the system geometry; LOS path, deterministic reflections and random reflections.

signal, the total received power is

$$
\begin{aligned}
P_{R x} & =P_{T x} \gamma(r) G_{T x}(0) G_{R x}(0) \\
& +\sum_{i=1}^{N} P_{T x} \gamma\left(R_{i}\right) G_{T x}\left(\alpha_{T x, i}\right) G_{R x}\left(\alpha_{R x, i}\right),
\end{aligned}
$$

where $P_{R x}$ is the received power, $P_{T x}$ is the transmitted power, $G_{T x}$ is the transmitter antenna gain, $G_{R x}$ is receiver antenna gain, $N$ is the number of multipath components, $R_{i}$ is the length of the $i$ th multipath component, $\alpha_{T x, i}$ is the angle of the $i$ th multipath component to the primary communication path, and $\alpha_{R x, i}$ is the same for the receiver. Notice that this is the mechanism that rejects the multipath components; the antenna pattern determines how wide field of view it has and the angle is dependent on where the reflection point is with respect to the main communication path of the $\mathrm{Rx}$ and $\mathrm{Tx}$. Path loss $\gamma(x)$ is defined as

$$
\gamma(x)=\frac{c^{2} \exp \left(-\kappa_{a} x\right)}{(4 \pi f x)^{2}},
$$

where $c$ is the speed of light, $x$ is the distance, $f$ is the frequency, and $\kappa_{a}$ is the molecular absorption coefficient, which can be estimated, e.g., like in [12]. And additional illustration of the simulation system's geometry is given in Fig. 3, where $p_{r}$ is the random reflection point, and $p_{b}$ is a blocking probability of a deterministic path.
The antennas in this work are assumed to be perfectly conical antennas with no side lobes. This is a good approximation in the high mmWave and $\mathrm{THz}$ band as the large path loss forces to utilize very high antenna gains where the main lobe gains are considerably higher to the side lobe levels. Additionally, they are easy to handle in simulation models and theoretical calculations. Such antennas would have a gain equivalent to

$$
G=\frac{1}{2 \pi\left(1-\cos \left(\theta_{\frac{1}{2}}\right)\right)}
$$

due to geometry of a cone and constant total radiated power, where $\theta_{\frac{1}{2}}$ is the antenna half beamwidth. It can be seen that the gain for a full sphere, i.e., for an isotropic antenna is $1 / 4 \pi$ to all directions. This is due to integration over the entire sphere equals $4 \pi$. Then, the full spherical integral over $G$ is always unit, i.e., the full Tx power.

\section{NUMERICAL RESULTS}

The simulations were run for a tight grid of Txs around the room, but with constant height of $120 \mathrm{~cm}$ to simulate a person holding the device. Although, the height is not a major issue here. We ran the simulation model for a $400 \times 600 \times 240$ $\mathrm{cm}^{3}$ room for this paper, but the results obtained for other room sizes agree with the models produced in this reference room. That is, also in the case we change the geometry. As a consequence, the absolute height of the device is not important as the overall behavior of the signal is dictated by the propagation paths and the angles of arrival to those, i.e., the reflection losses, and the antenna gains. The antenna (full) beamwidth was kept at $\pi / 16$ radians in the numerical results, corresponding to antenna gain of about $15 \mathrm{~dB}$ for rotationally symmetric antenna pattern assumed herein. The number of random reflection points distributed exponentially about the sides of the room varied from 25 to 100 . We also simulated the results for two different bandwidths, $10 \mathrm{GHz}$ and $100 \mathrm{GHz}$ with center frequency being $300 \mathrm{GHz}$. However, the bandwidth did not have any impact on the results and therefore the bandwidth is not considered below. Similarly, the antenna gains only scale the below results without having impact on the behavior of the path loss. Therefore, The actual received power according to the below models becomes

$$
P_{R x}=P_{T x} \gamma G_{T x}(0) G_{R x}(0),
$$

where $\gamma$ is channel gain that are given below for the LOS and NLOS communication cases. Finally, the blocking probability of the deterministic paths through walls, floor and ceiling was 0.5 . However, it should be noticed that the antenna gains render, e.g., the back wall invisible due to assumption of no side lobes.

The simulations results show that if the LOS path is available, the NLOS paths are either very weak compared to the LOS path or there are none available. This is natural due to large antenna gains to maintain proper signal levels. As a consequence, the following free space path loss model with 


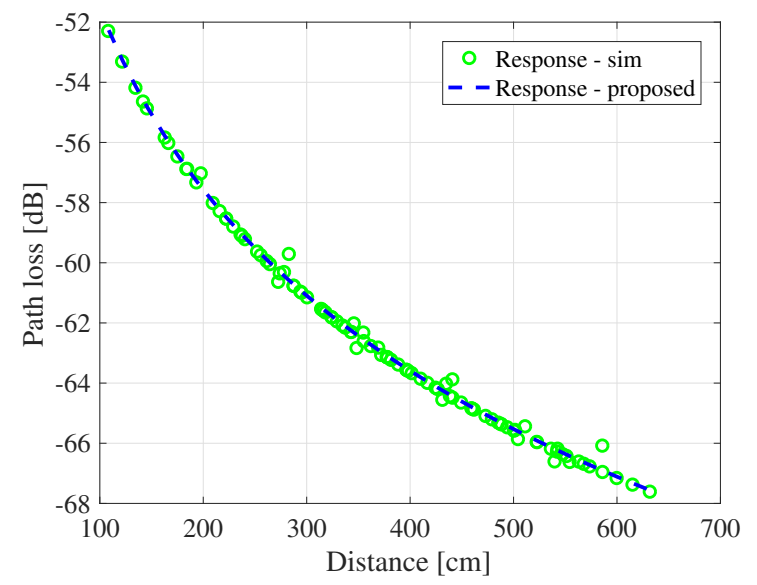

Fig. 4. Simulated and fitted path gain with about $30 \mathrm{~dB}$ total antenna gain as a function of distance for LOS case.

path loss exponent two describes the loss very accurately:

$$
\gamma_{\mathrm{LOS}}=6.41 \times 10^{-9} r^{-2},
$$

where $\gamma_{\text {LOS }}$ is the path gain of the channel and $r$ is the distance in meters between $\mathrm{Tx}$ and $\mathrm{Rx}$ along the primary signal path. Notice that this is not directly the free space path loss according to Friis transmission equation, but it also takes into account molecular absorption loss. The simulated path gains are shown in Fig. 4. The above free space model is given as dashed line. This shows a perfect fit between the simulation and proposed model.

Where things get more interesting is when there is no LOS path available. We model the reflected power by basic Fresnel equations and by assuming circularly polarized radiation [11]

$$
R\left(\theta_{i}\right)=\frac{1}{2}\left(R_{s}\left(\theta_{i}\right)+R_{p}\left(\theta_{i}\right)\right)
$$

where

$$
R_{s}\left(\theta_{i}\right)=\left|\frac{n_{1} \cos \left(\theta_{i}\right)-n_{2} \sqrt{1-\left(\frac{n_{1}}{n_{2}} \sin \left(\theta_{i}\right)\right)^{2}}}{n_{1} \cos \left(\theta_{i}\right)+n_{2} \sqrt{1-\left(\frac{n_{1}}{n_{2}} \sin \left(\theta_{i}\right)\right)^{2}}}\right|^{2}
$$

and

$$
R_{p}\left(\theta_{i}\right)=\left|\frac{n_{1} \sqrt{1-\left(\frac{n_{1}}{n_{2}} \sin \left(\theta_{i}\right)\right)^{2}}-n_{2} \cos \left(\theta_{i}\right)}{n_{1} \sqrt{1-\left(\frac{n_{1}}{n_{2}} \sin \left(\theta_{i}\right)\right)^{2}}+n_{2} \cos \left(\theta_{i}\right)}\right|^{2}
$$

are the reflectances of the perpendicular $\left(R_{s}\left(\theta_{i}\right)\right)$ and the parallel $\left(R_{p}\left(\theta_{i}\right)\right)$ signal components, $R\left(\theta_{i}\right)$ is the reflectance of circularly polarized signal, $\theta_{i}$ is the angle of incident, $n_{1}$ is the refractive index of air (assumed to be one), and $n_{2}$ is the refractive index of the material.

The Fresnel equation's geometry is calculated based on the geometry of the room, locations of the $\mathrm{Rx}$ and $\mathrm{Tx}$, the respective deterministic reflection point locations and the random reflection point locations. The simulation were run for three different refractive indices for reflective materials.

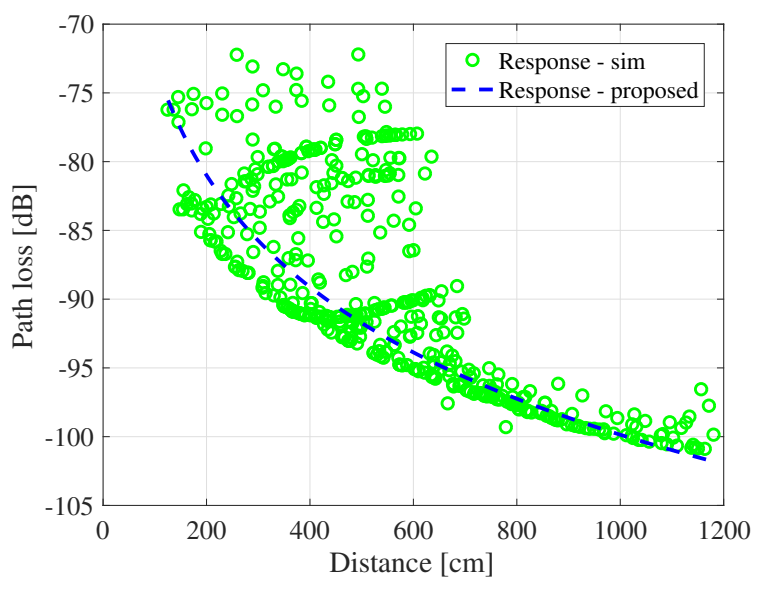

Fig. 5. Simulated and fitted path gain with about $30 \mathrm{~dB}$ total antenna gain as a function of distance for NLOS case with all the objects and walls having refractive index of 1.5 .

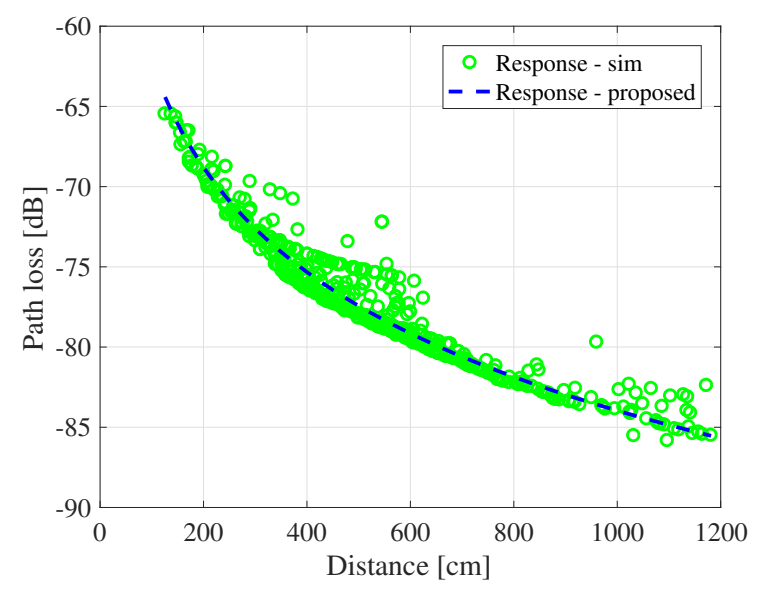

Fig. 6. Simulated and fitted path gain with about $30 \mathrm{~dB}$ total antenna gain as a function of distance for NLOS case with all the objects and walls having refractive index of 2.9 .

One case for refractive index of 1.5 , one for 2.9 and one for random refractive index varying between 1.5 and 2.9. These values were picked since our previous measurements have shown that many common indoor materials fall between these refractive indices around $300 \mathrm{GHz}$, but also around $1000 \mathrm{GHz}$ [11]. For instance, medium density fiber board (MDF) has a refractive index of about 1.5 at $300 \mathrm{GHz}$. On the other hand, glass as highly reflecting, but weakly penetrating material has a refractive index of about 2.9 at $300 \mathrm{GHz}$.

Figures 5 to 7 show the results for the above Fresnel equation assumptions. As expected, high refractive index suggests high reflected power (Fig 6). The low end the of the refractive indices allows larger penetration and less reflected power (Fig. 5). When the refractive index is random, we obtain the most plausible picture of the random signal propagation. However, also the channel gains are more random for any particular distance between the Tx and Rx. Notice that the distance here is the distance via the reflected path, not the Euclidean 


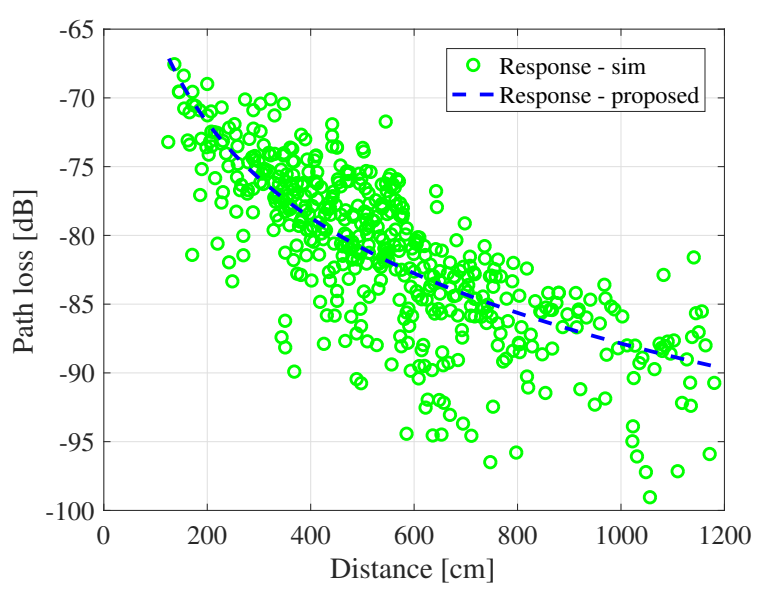

Fig. 7. Simulated and fitted path gain with about $30 \mathrm{~dB}$ total antenna gain as a function of distance for NLOS case with all the objects and walls having random refractive.

\section{distance.}

Based on the simulations, we derived the following model for NLOS communications by fitting to the simulation data:

$$
\gamma_{\mathrm{NLOS}}=1.19 \times 10^{-9} r^{-\alpha}
$$

where $\alpha$ are the path loss exponents obtained by simulations. Those were found as $\alpha=2.7,2.17$, and 2.3 for the refractive indices $n_{2}=1.5,2.9$, and random, respectively. Figures 5 to 7 show a very good fit of the simulation data to the proposed models.

The results here are give for a room shown in Fig. 1, but according to simulations on larger rooms, the proposed models remain accurate. The most interesting case among these is the random refractive index, as it gives the likely case with various materials present in a random indoor location. This particular case shows that an average NLOS path causes about $15 \mathrm{~dB}$ of additional loss to the LOS case. This is mainly attributed to the increased loss on the reflected paths. This is and expected result as the reflections will have less power than the LOS path.

The future work still requires some more estimation for proper random point distributions for various different environments. For instance, in office environment furniture are usually placed very much differently than in a usual home. Also, the exact distribution parameters require attention. But as a whole, simulations give a good overview of the general signal behavior. Measurements campaigns are also needed to further increase the credibility of the models.

\section{CONCLUSION}

In this paper we derived simple propagation models for indoor multipath environment by curve fitting to the simulation results. These simulations took into account deterministic reflections from the walls, floor and ceiling, and randomly picked reflection points that simulated objects in the space. The simulation results showed that for high gain antennas with LOS path available, the multipaths are irrelevant and the free space loss perfectly describes the expected path loss. If the NLOS paths are the primary communication channel, the path loss exponent is determined by the materials and the reflection loss they impose on the primary communication channel. Because of the highly directional antennas, the other multipaths keep the overall path loss exponent relatively close to the free space loss with additional loss being introduced by reflection loss. This additional loss was found to be around 15 $\mathrm{dB}$ in absolutely random environment. Whereas these models do not give answers to exact propagation of the radiowaves in the medium, they give an insight to the overall expected loss in a generic random indoor environment and the expected loss in those.

\section{ACKNOWLEDGEMENT}

This project (TERRANOVA) was supported by Horizon 2020, European Union's Framework Programme for Research and Innovation, under grant agreement no. 761794. This work was also supported in part by the Academy of Finland 6Genesis Flagship under grant no. 318927.

\section{REFERENCES}

[1] I. F. Akyildiz, J. M. Jornet, and C. Han, “Terahertz band: Next frontier for wireless communications," Elsevier Phys. Commun., vol. 12, pp. 1632, Sep. 2014.

[2] A.-A. A. Boulogeorgos, A. Alexiou, T. Merkle, C. Schubert, R. Elschner, A. Katsiotis, P. Stavrianos, D. Kritharidis, P. K. Chartsias, J. Kokkoniemi, M. Juntti, J. Lehtomäki, A. Teixeirá, and F. Rodrigues, "Terahertz technologies to deliver optical network quality of experience in wireless systems beyond 5G," IEEE Commun. Mag., vol. 56, no. 6, pp. 144-151, Jun. 2018.

[3] V. Petrov, J. Kokkoniemi, D. Moltchanov, J. Lehtomäki, Y. Koucheryavy, and M. Juntti, "Last meter indoor terahertz wireless access: Performance insights and implementation roadmap," IEEE Commun. Mag., vol. 56, no. 6 , pp. 158-165, June 2018.

[4] J. Kokkoniemi, J. Lehtomäki, V. Petrov, D. Moltchanov, and M. Juntti, "Frequency domain penetration loss in the terahertz band," in Proc. Global Symp. Milli. Waves, 2016, pp. 1-4.

[5] R. Piesiewicz, M. Jacob, M. Koch, J. Schoebel, and T. Kürner, "Performance analysis of future multigigabit wireless communication systems at $\mathrm{THz}$ frequencies with highly directive antennas in realistic indoor environments," IEEE J. Sel. Topics Quantum Electron., vol. 14, no. 2, pp. 421-430, Mar. 2008.

[6] S. Priebe and T. Kürner, "Stochastic modeling of $\mathrm{THz}$ indoor radio channels," IEEE Trans. Wireless Commun., vol. 12, no. 9, pp. 44454455, Sep. 2013

[7] S. Priebe, M. Kannicht, M. Jacob, and T. Kürner, "Ultra broadband indoor channel measurements and calibrated ray tracing propagation modeling at thz frequencies," J. Commun. Netw., vol. 15, no. 6, pp. 547-558, Dec. 2013.

[8] A. Moldovan, M. A. Ruder, I. F. Akyildiz, and W. H. Gerstacker, "LOS and NLOS channel modeling for terahertz wireless communication with scattered rays," in Globecom Workshops, 2014, pp. 388 - 392.

[9] F. Sheikh, N. Zarifeh, and T. Kaiser, "Terahertz band: Channel modelling forshort-range wireless communications in the spectral windows," IET Microw. Antennas Propag., vol. 10, no. 13, pp. 1435-1444, July 2016.

[10] C. Han, A. O. Bicen, and I. F. Akyildiz, "Multi-ray channel modeling and wideband characterization for wireless communications in the terahertz band," IEEE Trans. Wireless Commun., vol. 14, no. 5, pp. 2402-2412, May 2015.

[11] J. Kokkoniemi, J. Lehtomäki, and M. Juntti, "Reflection coefficients for common indoor materials in the terahertz band," in ACM NanoCom, 2018, pp. 1-6.

[12] J. M. Jornet and I. F. Akyildiz, "Channel modeling and capacity analysis for electromagnetic nanonetworks in the terahertz band," IEEE Trans. Wireless Commun., vol. 10, no. 10, pp. 3211-3221, Oct. 2011. 